\title{
Impacto de la valoración cardiovascular preoperatoria sobre las complicaciones cardiovasculares intraoperatorias y posoperatorias en cirugía de catarata
}

\section{Impact of preoperative cardiovascular assessment on intraoperative and postoperative cardiovascular complications in cataract surgery}

\author{
Dolores A. González-De la Mora ${ }^{1}$, Ernesto Terán-Martínez², Jazmín I. García Leonardo ${ }^{3 *}$ y \\ José R. Ponce-Martínez ${ }^{1}$

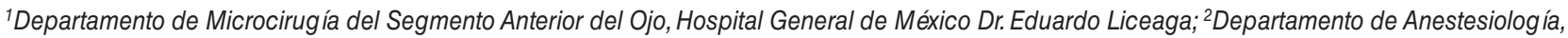 \\ Instituto Nacional de Ciencias Médicas y Nutrición Salvador Zubirán; ${ }^{3}$ Departamento de Oftalmología, Hospital General de México Dr. Eduardo \\ Liceaga; Ciudad de México, México
}

\section{Resumen}

Antecedentes: La catarata es la principal causa de ceguera reversible en todo el mundo; no obstante, en México, la tasa de cirugías realizadas se encuentra muy por debajo del ideal debido a la falta de acceso a servicios médicos y al precio del procedimiento quirúrgico. Aunque los exámenes prequirúrgicos se realizan siempre, no hay evidencia científica de que disminuyan los riesgos de complicaciones intraoperatorias y posoperatorias. Objetivo: Evaluar si existe relación entre las complicaciones cardiovasculares intraoperatorias y postoperatorias y la valoración cardiovascular preoperatoria sistemática en pacientes programados para cirugía de catarata en población mexicana. Método: Se incluyeron 327 pacientes operados de catarata en el servicio de oftalmología del Hospital General de México, en los que se buscó la presencia de complicaciones cardiovasculares y cerebrovasculares. Resultados: En el intraoperatorio no hubo ningún evento vascular mayor (infarto agudo al miocardio, accidente cerebrovascular o arritmias letales) independientemente de la presencia de comorbilidad, del tipo de anestesia y del tiempo de cirugía para pacientes con clasificación cardiovascular Goldman I y II. Tampoco se presentaron eventos cardiovasculares mayores en los siguientes 7 días. Conclusiones: La valoración cardiovascular preoperatoria tiene nula relación con la morbimortalidad intraoperatoria y posoperatoria de la cirugía de catarata.

Palabras clave: Catarata. Valoración preoperatoria. Riesgo cardiovascular. Infarto agudo de miocardio. Accidente cerebrovascular. Arritmias.

\section{Abstract}

Background: Cataract is the leading cause of reversible blindness worldwide; however, in Mexico, the rate of surgeries performed is far below ideal due to the lack of access to medical services and the price of the surgical procedure. Although preoperative examinations are always performed, there is no scientific evidence that they reduce the risks of intraoperative and postoperative complications. Objective: To assess whether there is a relationship between intraoperative and postoperative

Correspondencia:

*Jazmín I. García Leonardo

Excursionistas del D.F., Manzana 150, Lote 1572

Col. Lázaro Cárdenas

Fecha de recepción: 01-07-2020

C.P. 54189, Tlalnepantla, Estado de México, México

E-mail: jazmin.irais01 @ gmail.com

Fecha de aceptación: 16-03-2021

DOI: 10.24875/RMO.M21000171
Disponible en internet: 01-07-2021

Rev Mex Oftalmol. 2021;95(4):157-160

www.rmo.com.mx 0187-4519/๑ 2021 Sociedad Mexicana de Oftalmología. Publicado por Permanyer. Este es un artículo open access bajo la licencia CC BY-NC-ND (http://creativecommons.org/licenses/by-nc-nd/4.0/). 
cardiovascular complications and routine preoperative cardiovascular assessments in patients scheduled for cataract surgery in a Mexican population. Method: 327 patients undergoing cataract surgery were included in the ophthalmology service of the Hospital General de México, in which the presence of cardiovascular and/or cerebrovascular complications was evaluated. Results: During the intraoperative period, there were no major vascular events (acute myocardial infarction, cerebrovascular accident, or lethal arrhythmias) regardless of the presence of comorbidity, the type of anesthesia, and the time of surgery for patients with Goldman I and II cardiovascular classification. There were also no major cardiovascular events in the next 7 days. Conclusions: Preoperative cardiovascular assessment has no relationship with intraoperative and postoperative morbidity and mortality of cataract surgery.

Key words: Cataract. Preoperative testing. Cardiovascular risk. Acute myocardial infarction. Stroke. Arrhythmia.

\section{Introducción}

La opacidad del cristalino es la principal causa de ceguera reversible en las personas mayores de 50 años, pero a pesar de la alta incidencia de catarata en México, la tasa de cirugías realizadas se encuentra muy por debajo del ideal debido principalmente a la falta de acceso a servicios médicos y al precio del procedimiento quirúrgico, estimando un costo de hasta 150 millones de dólares anuales solo en las pruebas preoperatorias sistemáticas ${ }^{1-4}$.

Aunque la cirugía de catarata es considerada de bajo riesgo, en México todos los pacientes deben ser evaluados para identificar la presencia de factores de riesgo asociados a complicaciones, por lo que de forma sistemática se solicitan un hemograma completo y un electrocardiograma para los pacientes de 65 años o más, independientemente de su estado de salud; otras pruebas, como radiografía de tórax, estudios de coagulación sanguínea y análisis de orina, también son ordenados con frecuencia 5 .

Hasta ahora, los estudios realizados no han demostrado que la evaluación prequirúrgica disminuya el riesgo de complicaciones sistémicas intraoperatorias y posoperatorias, ni que aumente la seguridad de la cirugía, por lo que en algunos países desarrollados los pacientes de bajo riesgo se programan de forma directa a cirugía omitiendo los "exámenes de rutina", y con ello se ha logrado reducir de manera significativa los costos de la cirugía de catarata y aumentar la eficiencia del sistema ${ }^{6,7}$.

Debido a la incertidumbre sobre la efectividad de estas pruebas, el presente estudio tiene la finalidad de evaluar si las pruebas médicas y la valoración cardiovascular sistemática antes de la cirugía de cataratas reducen la tasa de complicaciones cardiovasculares intraoperatorias y posoperatorias en la población mexicana.

\section{Método}

Se realizó un estudio analítico, descriptivo, prospectivo y observacional no experimental en el periodo comprendido entre marzo de 2017 y febrero de 2018 en el Hospital General de México. Se incluyeron pacientes de cualquier edad y sexo diagnosticados de catarata, con estudios de laboratorio y valoración cardiovascular de acuerdo con la escala de Goldman que fueron sometidos a tratamiento quirúrgico. Los pacientes sin valoración cardiovascular, catarata congénita, cataratas traumáticas y aquellos en los que se realizó cirugía de catarata combinada con algún otro procedimiento fueron excluidos del estudio.

Los pacientes firmaron un consentimiento informado de acuerdo con la Ley General de Salud y la Declaración de Helsinki. El presente estudio fue evaluado y aprobado por el comité de ética del Hospital General de México con clave de registro DIR/17/102/3/012.

Mediante interrogatorio directo y revisión de expedientes se obtuvieron los siguientes datos clínicos y demográficos: edad, sexo, comorbilidad y riesgo cardiovascular en la escala de Goldman, técnica quirúrgica, grado académico del cirujano y tipo de anestesia empleada.

Se evaluó la presencia de complicaciones cardiovasculares, como isquemia, infarto al miocardio y accidente vascular cerebral, para lo cual el servicio de anestesiología llevó a cabo vigilancia intraoperatoria registrando la presión arterial sistólica (PAS), diastólica (PAD) y media (PAM) en intervalos de 10 minutos, y el ritmo cardiaco mediante registro electrocardiográfico continuo. Se evaluó el estado neurológico antes y después de la intervención mediante la National Institute of Health Stroke Scale hasta el egreso del servicio de oftalmología. Dichos estudios se repitieron una semana después de la cirugía.

Los datos se analizaron mediante la prueba $t$ de Student para muestras independientes en las variables 
cuantitativas, con un intervalo de confianza del 95\%. Las variables categóricas se analizaron por medio del paquete estadístico STATA 14 para Windows. Todos los análisis se realizaron considerando significativo un valor de $p<0.05$.

\section{Resultados}

Se estudiaron 327 pacientes, el $58 \%$ mujeres y el $42 \%$ hombres, con una edad media de 66 años (rango: 26 a 94$)$.

En el $82 \%$ de los pacientes se realizó facoemulsificación, mientras que el $18 \%$ restante fue sometido a extracción extracapsular de catarata.

El $68 \%$ de los pacientes fueron operados por residentes en formación de microcirugía del segmento anterior y el $32 \%$ restante por el médico adscrito al servicio de oftalmología.

Dentro del perfil clínico de los pacientes, el 73.7\% tuvo una enfermedad sistémica asociada; la más frecuente fue hipertensión arterial en el $55.6 \%$, seguida de diabetes mellitus tipo 2 en el $45.8 \%$. El 2.14\% de los pacientes tuvieron tolerancia alterada a los hidratos de carbono (Tabla 1).

En la valoración cardiovascular, el $58 \%$ se encontró en categoría II y el $42 \%$ en categoría I de la escala de Goldman.

La modalidad anestésica empleada fue bloqueo retrobulbar más anestesia tópica en el $62 \%$, anestesia intracameral en el $21 \%$, bloqueo retrobulbar en el $16 \%$, sedación intravenosa en el $0.2 \%$ y anestesia general en el $0.1 \%$ (Fig. 1).

Para la PAS se obtuvo una diferencia entre los valores inicial y final de la cirugía de $0.36 \mathrm{mmHg}$, con t de Student de 0.7; para la PAD, el valor t de Student fue de 0.9 , con una diferencia de $0.06 \mathrm{mmHg}$; y para la PAM, $\mathrm{t}$ de Student de 1.0 con una diferencia de $-0.01 \mathrm{mmHg}$.

En el intraoperatorio no se presentó ningún evento cardiovascular mayor (infarto agudo al miocardio, accidente cerebrovascular o arritmias letales). Tampoco se presentaron eventos cardiovasculares dentro de los 7 días posoperatorios.

\section{Discusión}

La guías de evaluación y manejo cardiovascular en pacientes para cirugía no cardiaca de la American Heart Association y de la European Society of Cardiology establecen que la cirugía de catarata tiene un riesgo menor del $1 \%$ para complicaciones cardiovasculares mayores. El presente estudio demuestra
Tabla 1. Frecuencia de comorbilidad

\begin{tabular}{|l|c|}
\hline Comorbilidad & Porcentaje (n) \\
\hline Hipertensión arterial sistémica & $55.6 \%(182)$ \\
\hline Diabetes mellitus tipo 2 & $45.8(150)$ \\
\hline Tolerancia a los hidratos de carbono alterada & $2.14 \%(7)$ \\
\hline Cardiopatía isquémica + stent & $0.3 \%(1)$ \\
\hline Antecedente de arritmia cardiaca & $0.6 \%(2)$ \\
\hline Accidente vascular cerebral & $0.6 \%(2)$ \\
\hline Ninguna & $26.2 \%(86)$ \\
\hline
\end{tabular}

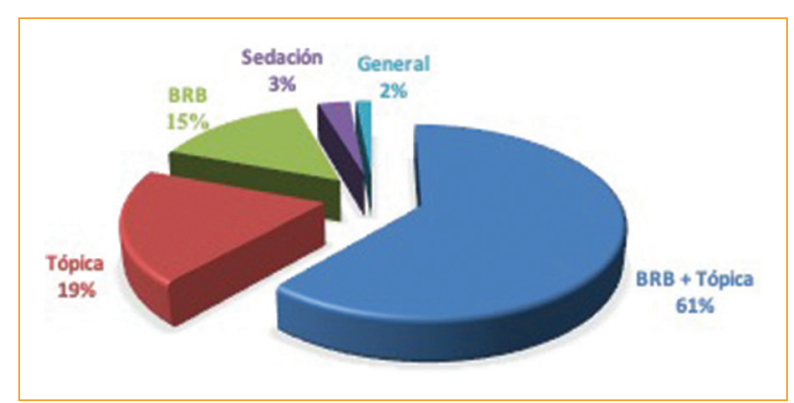

Figura 1. Tipo de anestesia empleada. BRB: bloqueo retrobulbar.

que la incidencia de complicaciones cardiovasculares es todavía más cercana a cero.

No se encontró que el sexo, la edad o la presencia de comorbilidad concediera algún factor de riesgo para la presencia de complicaciones cardiovasculares.

No hubo diferencia entre el tipo de anestesia empleada y la presencia de complicaciones cardiovasculares, por lo que la modalidad anestésica parece no conferir factor protector alguno ni agregar factor de riesgo para complicaciones cardiovasculares.

A pesar de que los cirujanos en formación emplean un mayor tiempo quirúrgico, esto no confirió un riesgo para la presencia de complicaciones cardiovasculares.

Es importante mencionar que el índice de Goldman es la única escala cardiovascular utilizada en la valoración preoperatoria del Hospital General de México, y una de las más frecuentes utilizadas para la población mexicana en general, y que la cirugía de catarata se realiza principalmente de forma ambulatoria en el Hospital General de México, motivo principal por el que los pacientes incluidos en este estudio presentaban una valoración de Goldman I y II, ya que los pacientes con Goldman IV suelen tener un importante deterioro 
del estado general que impide que acudan a una revisión oftalmológica bajo lámpara de hendidura. No obstante, diversos estudios han demostrado que esta escala es poco específica para predecir complicaciones cardiovasculares, por lo que en los últimos años se han descrito otras, como P-POSSUM, GUPTA y NSQIP, que de acuerdo con los estudios realizados muestran resultados clínicos y estadísticos que pudieran ser aplicados a la población mexicana.

Nuestro estudio es el primero en realizar una revisión para evaluar la utilidad de las pruebas médicas sistemáticas antes de la cirugía de cataratas en la tasa de complicaciones perioperatorias en población mexicana con comorbilidad.

Sugerimos que los pacientes programados para cirugía de catarata sean valorados y estratificados en la consulta externa de oftalmología de acuerdo con las escalas internacionales actuales para riesgo cardiovascular, y que de esta manera se identifique a los pacientes que ameritan realmente una valoración extendida por medicina interna, cardiología o anestesiología.

\section{Conclusiones}

Presentamos el primer estudio enfocado en evaluar si las pruebas prequirúrgicas sistemáticas son útiles para reducir la tasa de complicaciones perioperatorias en la cirugía de cataratas en población mexicana, concluyendo que, independientemente de la presencia de comorbilidad, del tipo de anestesia y del tiempo de cirugía empleado, el riesgo cardiovascular asociado a la cirugía de catarata permanece bajo $(0 \%)$ para los pacientes con clasificación cardiovascular Goldman I y II, por lo que los médicos oftalmólogos deberíamos tener la capacidad para evaluar y estratificar en la consulta externa de oftalmología qué pacientes ameritan realmente una valoración extendida por medicina interna, cardiología o anestesiología, lo cual podría realizarse de manera efectiva mediante las nuevas escalas de valoración preoperatoria con el objetivo de disminuir los altos costos de una valoración preoperatoria sistemática, evitar el retraso en la programación de la cirugía y disminuir la tasa de ceguera reversible por catarata.

\section{Financiación}

El presente artículo se realizó con recursos del servicio de Oftalmología en el Hospital General de México "Dr. Eduardo Liceaga".

\section{Conflictos de intereses}

Los autores del presente artículo declaramos no tener ningún tipo de conflicto de intereses, así como ninguna relación económica, personal ni política.

\section{Responsabilidades éticas}

Protección de personas y animales. Los autores declaran que para esta investigación no se han realizado experimentos en seres humanos ni en animales.

Confidencialidad de los datos. Los autores declaran que han seguido los protocolos de su centro de trabajo sobre la publicación de datos de pacientes.

Derecho a la privacidad y consentimiento informado. Los autores declaran que en este artículo no aparecen datos de pacientes.

\section{Bibliografía}

1. Agarwal A, Kumar DA. Cost-effectiveness of cataract surgery. Curr Opin Ophthalmol. 2011;22:15-8.

2. Lee CM, Afshari NA. The global state of cataract blindness. Curr Opin Ophthalmol. 2017;28:98-103.

3. Lansingh VC, Resnikoff S, Tingley-Kelley K, Nano ME, Martens M, Silva JC, et al. Cataract surgery rates in Latin America: a four-year longitudinal study of 19 countries. Ophthalmic Epidemiol. 2010;17:75-81.

4. Batlle JF, Lansingh VC, Silva JC, Eckert KA, Resnikoff S. The cataract situation in Latin America: barriers to cataract surgery. J Ophthalmol. 2014;158:242-50.

5. Committee on Standards and Practice Parameters; Apfelbaum JL, Connis RT, Nickinovich DG, American Society of Anesthesiologists Task Force on Preanesthesia Evaluation; et al. Practice advisory for preanesthesia evaluation: a report by the American Society of Anesthesiologists Task Force on Preanesthesia Evaluation. Anesthesiology. 2002;96:485-96.

6. Keay L, Lindsley K, Tielsch J, Katz J, Schein O. Routine preoperative medical testing for cataract surgery. Cochrane Database Syst Rev. 2012;(3):3-6.

7. Schein OD, Katz J, Bass EB, Tielsch JM, Lubomski LH, Feldman MA, et al. The value of routine preoperative medical testing before cataract surgery. Study of Medical Testing for Cataract Surgery. N Engl J Med. 2000;342: $168-75$ 